\title{
Ultra Grain Refinement in a Plain Carbon Steel by Means of Warm Torsion Testing
}

\author{
Welbert Ribeiro CALADO and Ronaldo Antônio Neves Marques BARBOSA \\ Department of Metallurgical and Materials Engineering, Federal University of Minas Gerais, 6627 Antônio Carlos Avenue, \\ Group 2, Room 2230, Belo Horizonte, Minas Gerais, CEP 3409-1815, Brazil. E-mail: welbert.ribeiro@nuclep.gov.br
}

(Received on March 8, 2010; accepted on June 18, 2010)

\begin{abstract}
Ultra grain refinement of a plain carbon SAE-AISI 1010 steel has been obtained by means of warm torsion testing. Tests were conducted at $500^{\circ} \mathrm{C}$, constant strain rate of $0.1 \mathrm{~s}^{-1}$ and total equivalent strain of $0.8,1.6$, 4.0 and 5.6. The average ferritic grain size obtained was smaller than $0.5 \mu \mathrm{m}$. The steel microstructure consisted of elongated ferrite grains and fragmented and spheroidized cementite particles, similar to the microstructures found in carbon steel severely plastic deformed, like in the ARB and ECAP processes. The average ferritic grain size is reasonably predicted by the Hall-Petch equation. The mechanisms by which ultra grain refinement has been achieved here has been attributed to dynamic recovery and continuous dynamic recrystallization of the ferrite during warm plastic deformation. Warm torsion testing proved to be a suitable tool to study grain refining mechanism through severe plastic deformation.
\end{abstract}

KEY WORDS: ultrafine grained steel; torsion testing; dynamic recovery; continuous recrystallization; severe plastic deformation.

\section{Introduction}

There has been growing interest in the development of new routes to produce metals with ultrafine grained (UFG) structure. This interest is attributable to the inherent and exclusive properties of metals with UFG: increases in the strength without deterioration of toughness. The methods of processing ultrafine grained metals are divided into two main groups: one based on advanced thermomechanical treatments and the other based on severe plastic deformation. ${ }^{1,2)}$ The first method combines phase transformation and the application of controlled deformation to obtain grain refinement. The second method, based on severe plastic deformation, uses the application of large cold or warm deformations to obtain UFG. The main techniques for the production of UFG metals by severe plastic deformation are: Equal Channel Angular Pressing (ECAP), ${ }^{3)}$ High Pressure Torsion ${ }^{4}$ and Accumulative Roll-Bonding ${ }^{5)}$ (ARB). The latter seems to be the most likely to be used as an application at industrial scale. This technique consists of applying high deformation on sheets or strips of metal through successive cold or warm roll-bonding cycles, under high friction conditions, i.e., without lubrication. ${ }^{3,6)}$ Grain refinement in the ARB and ECAP processes is attributed to dynamic recovery and continuous dynamic recrystallization. ${ }^{7-9)}$ Despite the great importance of ARB, most papers on this subject deals with metals and alloys of low yield strength, such as aluminum, copper and interstitial free steel. This limitation can be attributed to the difficulty of achieving the large loads required during laboratory scale rolling to deform more resistant materials such as plain car- bon steels and stainless steels. Thus, the development of a methodology capable of simulating the ARB becomes an interesting alternative. Warm torsion tests may be a promising candidate for this simulation. This technique is simple and has been used successfully in the simulation of hot rolling of carbon steels for many years. ${ }^{10)}$ This study aims at investigating and validating the effectiveness with which torsion tests can be used for the study grain refining mechanism through severe plastic deformation applying the same parameters as for of the ARB process.

\section{Experimental Procedure}

A SAE-AISI 1010 plain carbon steel was used in the severe plastic deformation torsion test. This composition was selected because seldom are the studies reporting on the development of UFG for carbon steels with C contents greater than $0.1 \%$ in the literature and because there are also evidences that cementite can improve the ductility of the UFG steels. ${ }^{11,12)}$

Specimens for torsion testing were machined with diameter and useful length of 6.4 and $17.0 \mathrm{~mm}$, respectively. The specimens were heat treated to homogenize the microstructure at a $900^{\circ} \mathrm{C}$ for $5 \mathrm{~min}$ in a radiant infrared furnace. The torsion tests were performed on an MTS servohydraulic machine with torque cell capacity of $250 \mathrm{Nm}$ and TestStarII ${ }^{\circledR}$ interface. Samples were pre-heated to test temperature with protective argon atmosphere. The samples were heated to test temperature at $3^{\circ} \mathrm{Cs}^{-1}$, followed by a soaking period of $5 \mathrm{~min}$ after which deformation of true strain equals 0.8 was applied. Right after the deformation, 
the samples were cooled to room temperature inside the furnace. This procedure was repeated five times until a total equivalent strain of 4.0. Tests were carried out at $500^{\circ} \mathrm{C}$ and at a strain rate of $0.1 \mathrm{~s}^{-1}$. Additional tests were conducted applying total deformation of $0.8,1.6$ and 5.6 to evaluate the microstructure evolution and the effect of deformation on the average ferritic grains size.

Metallography was carried out in a polished section parallel to the sample axial direction just below the surface at the sample length. Etching was carried out with $2 \%$ nital for $8 \mathrm{~s}$. Analyses were made using optical and scanning electron microscopy. Average grain sizes were obtained by counting at least 200 grains giving an average error equals to $10 \%$. Vickers microhardness measurements were performed using $500 \mathrm{gf}$ load penetration.

\section{Results}

\subsection{Torsion Tests}

Figure 1 shows the equivalent stress-strain curves obtained from torsion tests conducted at $500^{\circ} \mathrm{C}$ and $0.1 \mathrm{~s}^{-1}$ strain rate. The maximum stress deformation was approximately $400 \mathrm{MPa}$ soon in the early stages and decreased to approximately $390 \mathrm{MPa}$ when the tests were finished. This behavior was not observed in the first pass (curve a). The different aspect of the curve of the first torsion pass can be correlated with the initial sample hardening during the plastic deformation.

Figure 2 shows equivalent stress-strain curves of specimens tested in torsion until fracture. One of the curves refers to a sample deformed up to failure in the state of as annealed, at $900^{\circ} \mathrm{C}$ for $5 \mathrm{~min}$. The other curve refers to the specimen tested in five pass torsion test. It can be seen that the stresses at an equivalent strain of 0.002 were 325 and $600 \mathrm{MPa}$ respectively for the annealed specimen and for the specimen processed in the five pass experiment. The specimen in the as annealed state fractured at a deformation of 3.9 and a flow stress level of $700 \mathrm{MPa}$. The sample tested in five torsion pass reached a total strain of 1.3 and flow stress of $750 \mathrm{MPa}$. It is important to note that both samples strain hardened significantly during testing. This is of importance because it is well known in the literatures that the low ductility usually found in ultrafine grained metals is due their low strain hardening ability. ${ }^{14,15)}$ In this way, the present strain hardening in the SAE 1010 carbon steel can contribute for a better ductility.

\subsection{Analysis of the Microstructures Obtained}

Figure 3 shows the microstructure for the sample in the as annealed state condition. It consists of equiaxed ferrite grains and perlite colonies, with average ferritic grain size of $13.7 \mu \mathrm{m}$. After torsion at $500^{\circ} \mathrm{C}$, it is no longer possible to distinguish the grains using optical microscopy. As it can be seen, the microstructure displays dark lines which are probably cementite lamellae of pearlite probably fragmented and aligned during deformation. This microstructure closely resembles the images obtained for plain carbon steels severely warm deformed, as presented in the work by Song et al. studying the microstructural evolution of C-Mn steel severely deformed by compression test. ${ }^{13)}$ Figure 4 shows the image obtained with scanning electron mi-

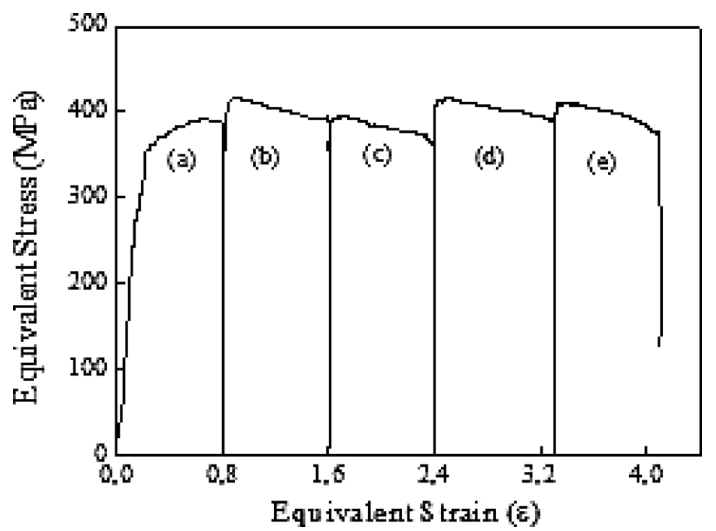

Fig. 1. Equivalent stress-strain curves for SAE 1010 steel tested in torsion at $500^{\circ} \mathrm{C}$ and $0.1 \mathrm{~s}^{-1}$ strain rate deformation.

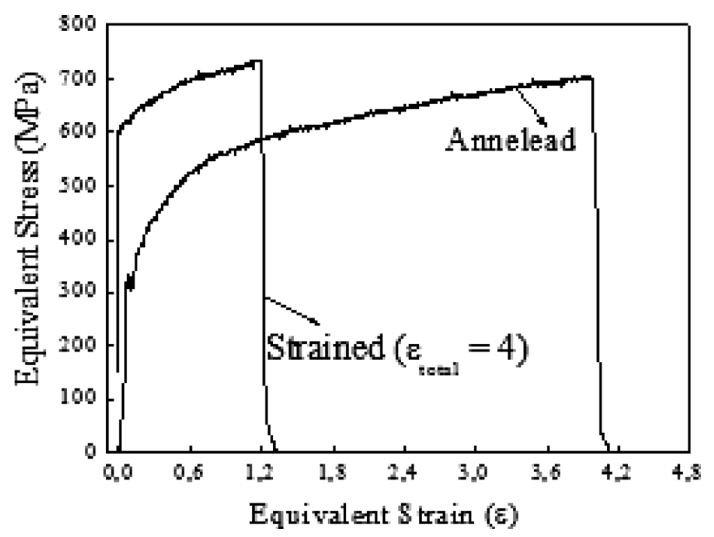

Fig. 2. Comparison of stress-strain curves for SAE 1010 steel samples deformed until fracture in the annealed state condition and after five passes torsion test at $500^{\circ} \mathrm{C}$.

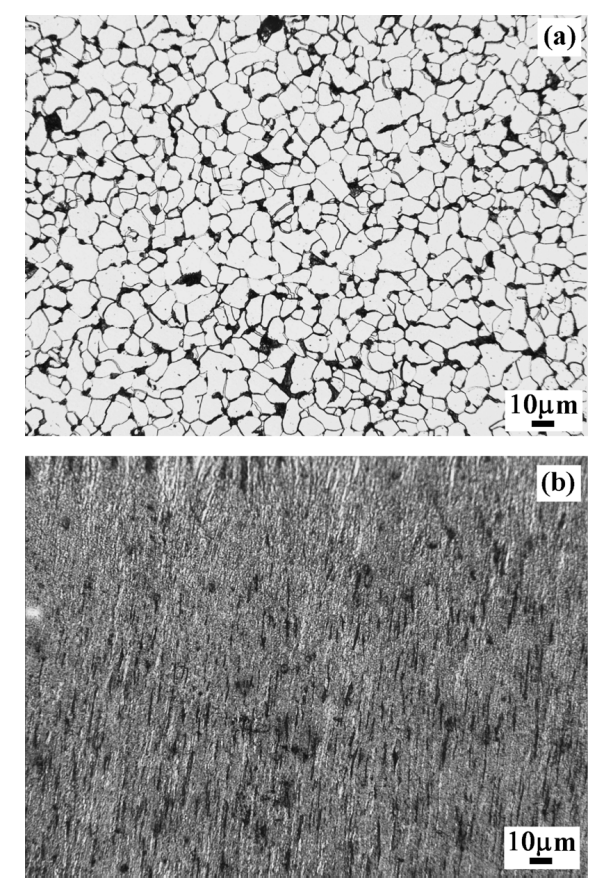

Fig. 3. Optical microscopy of SAE 1010 steel: (a) annealed state, (b) after torsion test.

croscopy for the same sample.

The average ferritic grain size obtained after the torsion tests was $0.4 \mu \mathrm{m}$ with a standard deviation of 0.05 . Some fragmented cementite lamellae of the original pearlite 


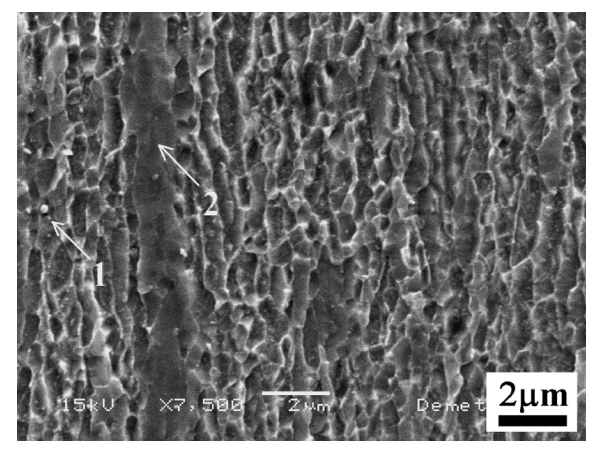

Fig. 4. Scanning electron microscopy of SAE 1010 steel after torsion tests at $500^{\circ} \mathrm{C}, \varepsilon=4.0$.

colonies are clearly visible. The ferritic grains are slightly elongated and some are more or less equiaxed. There are also some spheroidized cementite particles within some grains as pointed by the white arrow 1 . The figure also shows the presence of some elongated coarsened grains, as indicated by the white arrow 2 , with some faint traces that can be low angle grains boundaries.

Interrupted torsion tests were performed applying total logarithmic strain of $0.8,1.6$ and 5.6, corresponding to 1,2 and 7 passes of deformation, respectively. The idea was to evaluate the microstructural evolution and mechanisms of grain refining and the effect of total accumulated strain on the average ferritic grain size. Figure $\mathbf{5}$ shows the presence of low angle grain boundaries within the ferritic grains after a torsion pass deformation displayed as lines with clear contrast. At the same time, these ferrite grains are beginning to be stretched and some alignment and fragmentation of perlite colonies are also visible. In the sample processed by two torsion passes, it can still be seen the presence of some lighter contours that can be low angle grains boundaries within a large elongated ferrite grain; region bounded by the white circle. After deformation of 0.8 the average size of ferritic grain suffered only a slight reduction compared to the grain size of the sample in the as annealed state. Figure 6 summarizes the reduction of average grain size with equivalent accumulated strain.

\section{Discussion}

\subsection{Torsion Testing}

The equivalent stress-strain curves reported here are characteristic of metallic materials that undergo dynamic recovery during plastic deformation as seen in Fig. 1. As it is well known, ferrite, a bcc structure, has a high stacking fault energy, so dislocations easily undergoes heat-activated cross-slip and polygonization forming a cellular structure. Curve (a) in Fig. 1 corresponds to the first torsion deformation pass of a total of 5 at $500^{\circ} \mathrm{C}$. In this curve the steel strain hardens until deformation for this pass is interrupted. The initial stress at the second pass, curve (b), is approximately equal to the stress at the end of the previous deformation, reaching a peak at the start of flow and decreasing continuously until the end of the pass. All the other curves, (c), (d) and (e) showed the same behavior as for curve (b). This small reduction in the plastic flow stress is due to dynamic recovery that occurs during testing, this recovery is favored by moderate temperature of $500^{\circ} \mathrm{C}$ maintained
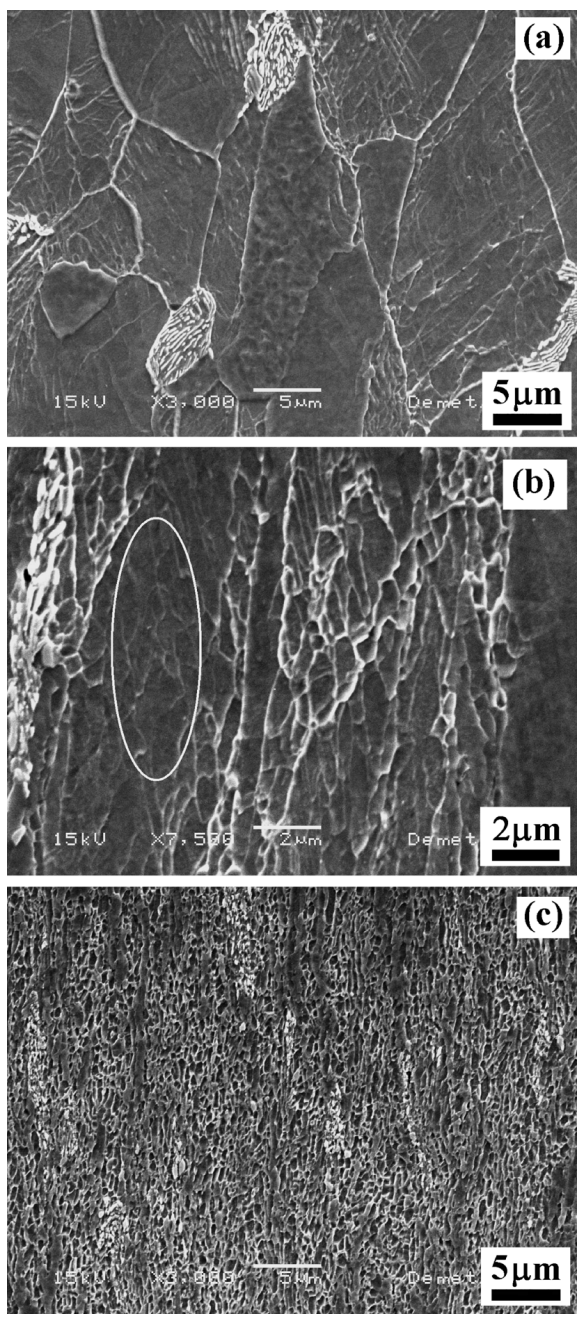

Fig. 5. Microstructure evolution with the equivalent accumulated strain: (a) $\varepsilon=0.8$, (b) $\varepsilon=1.6$, (c) $\varepsilon=5.6$.

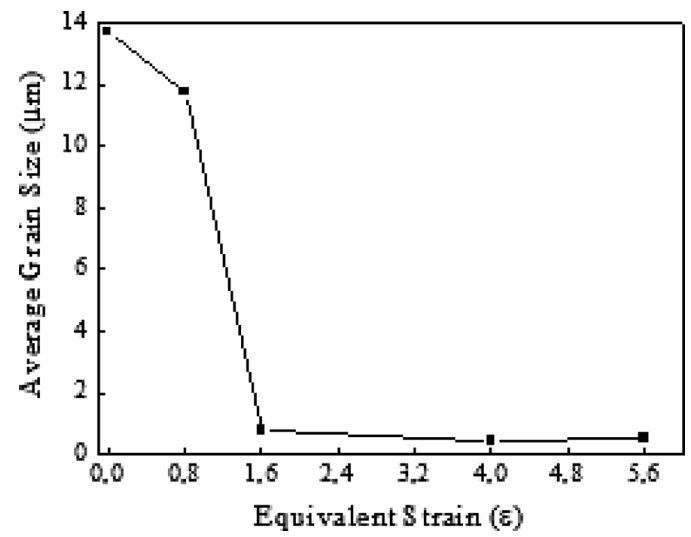

Fig. 6. Ferritic average grain size evolution with the equivalent accumulated strain.

throughout the torsion. As expected, there is no static recrystallization during the cooling of the specimen after each pass and in the same way there was no dynamic recrystallization during the experiment.

\subsection{Microstructure and Grain Refining Mechanisms}

From the microstructure standpoint, severe plastic deformation by means of warm torsion testing looks quite satisfactory, that is, the grains obtained are morphologically similar to those obtained by other researchers working with 
conventional process like ECAP and ARB. ${ }^{14,15)}$ This is also an indication that the key mechanisms for grain refinement are basically the same, as will be discussed in next paragraphs.

UFG metals were mostly obtained via severe plastic deformation. In this process, UFGs were formed by the subdivision of original grains in low angle grain boundaries in the early stages of deformation, followed by recovery and migration of these low angle boundaries to form high angle grains boundaries in next stages of plastic deformation. This is the case, specifically of Lee et al. that showed that the shear stress was of great importance in the formation of fine grains. ${ }^{16)}$

In this work, after the first torsion pass, low angle boundaries were formed within the original ferrite grain as seen in Fig. 5. The average grain size remained nearly unchanged if the experimental deviations are considered according to Fig. 6. Therefore, the first torsion pass promotes the formation of low angle grains boundaries, only. The grain size is then effectively reduced from the second pass onwards. This is consistent with the appearance of curve (a) at Fig. 1 that corresponds to the first torsion pass, where the steel strain hardens without recovery until the end of the test.

According to Fig. 5, low angle grain boundaries are gradually transforming into high angle grain boundaries. At the same time, very small grains can be already observed. Curve (b) at Fig. 1 indicates that from the second torsion pass the steel undergoes recovery only in the course of the deformation, corresponding well to the presence of the first ultrafine grains after 1.6 logarithmic strain. It can be here speculated that from the first to the second torsion deformation the recovery process together with a possible migration of low angle grain boundaries were acting in the formation of ultrafine grains. According to Fig. 6, the average grain size is greatly reduced after the second torsion pass with a strain accumulation of 1.6. After the fifth and seventh passes, accumulated strain of 4.0 and 5.6 respectively, there was no significant grain refinement and the curve reaching a plateau. This may mean that no significant large strain would be necessary to produce very fine grains in steels. This, on the other hand, could also mean that industry production of UFG may not be as far fetched as usually thought.

Consistently, torsion testing have shown that the higher the deformations the smaller the average ferritic grain size. However, this deformation apparently needs not to be extremely high. On the contrary, relatively small deformations generated very fine grains. Perhaps, the increase in the total deformation only contributes to a greater fraction formation of high angle grains boundaries. ${ }^{15)}$

In the torsion test of cylindrical solid specimen geometry, the shear stress varies linearly along specimen radius, being nil in the center and maximum at the surface. Direct consequence of this is that the average grain size of a specimen deformed by torsion varies along the radius, presenting the lowest values in the region near the surface. According to Lee et $a l .{ }^{16)}$ and Costa et al. ${ }^{7)}$ the shear stress in the ARB process greatly affects grain refinement, that is, it is more effective in region where the shear stress is maximum. In this way, the surface layers of a hot strip which is in direct contact with the rollers during deformation tend to present average grain size smaller than in the center of the band. It is clear then why there is a similarity between microstructures obtained from plastic deformation in torsion and from ARB processing. In the same way, as in the ARB and ECAP processes, the grain refinement in the specimens tested by torsion seems to be due to the process of subdividing the original grains in low angle grain boundaries and the continuous transformation of theses low angles grains in high angle grains boundaries trough dynamic recovery process and short distance migration.

\subsection{Mechanical Properties}

The equivalent stress required to start plastic deformation on the SAE 1010 steel tested in five passes torsion test is considerably higher than the stress to beginning plastic flow in the sample annealed only, namely $171 \%$ higher, Fig. 2. Although the sample tested in torsion fractured at a lower stress than that for the annealed sample, there is strong evidence that the ductility of these samples can be relatively high when deformed at standard tensile tests. The present strain hardening obtained during the torsion test may be due the presence of spheroidized cementite particles acting as sources and barriers pinning the dislocations. ${ }^{19)}$

Figure 7 shows the evolution of the Vickers hardness of the SAE 1010 steel as a function of the total equivalent strain in torsion tests performed at $500^{\circ} \mathrm{C}$ and the steel in the annealed condition.

Despite of the small reduction in the average grain size after the first pass deformation (Fig. 6), the microhardness increased sharply, there after. The hardness increased from about $110 \mathrm{HV}$ in the annealed condition to approximately $180 \mathrm{HV}$ after deformation of just 0.8. This significant increase in microhardness may be associated with the emergence of low angle grains boundaries in the first torsion pass. After the second torsion pass the microhardness displayed a small increase, reaching a value of approximately $200 \mathrm{HV}$ only, despite the considerable grain refinement. As expected, the hardness continues to increase steadily with the degree of plastic deformation.

This continuous increase in the microhardness with the accumulated strain after the second pass deformation can be directly linked to the increasing fraction of high angle grains boundaries, since, according to Fig. 6, the average grain size remains virtually unchanged. This behaviour is identical to the Vickers hardness curve obtained by Krallics

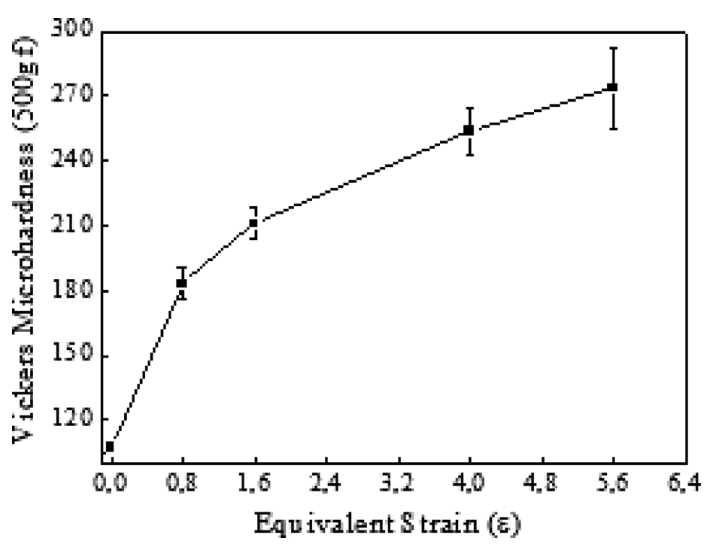

Fig. 7. SAE 1010 steel microhardness evolution with the total plastic deformation. 


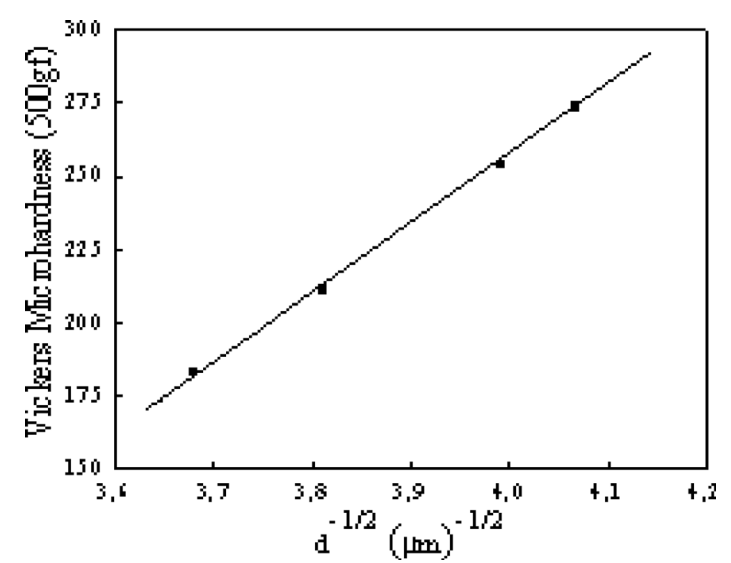

Fig. 8. SAE 1010 steel microhardness modeling through Hall-Petch equation.

and Lenard ${ }^{17)}$ studying the ARB processing of an IF steel. They showed that the hardness of IF steel increases with the number of processing cycles, and correlated this hardness with the number of layers obtained after each cycle. Cherukuri et al. ${ }^{18)}$ also obtained a hardness profile similar to that of Fig. 8 processing pure aluminum through the $\mathrm{ARB}$ process at room temperature.

Figure 8 shows a microhardness graph as a function of the inverse of the square root of the average ferritic grain size. The curve obtained is a straight line through all points. The data shown are adequately modeled by the Hall-Pech relationship, in agreement with report by Costa et al. ${ }^{7)}$ studying the ARB processing of an IF steel.

\section{Conclusions}

In the present study, ultra grain refinement of a plain carbon steel was studied by means of warm torsion testing applying the same parameters usually employed for ARB processing. The steel samples were tested at $500^{\circ} \mathrm{C}$ and $0.1 \mathrm{~s}^{-1}$ strain rate. The results obtained can be summarized as follows:

(1) The measured ferritic average grain size was smaller than $0.5 \mu \mathrm{m}$ after five passes torsion test.

(2) A total equivalent deformation of 1.6 is sufficient to promote ferrite grain refinement. Increasing total deformation beyond this limit adds little to grain refinement. The grain size stays practically the same, however increasing deformation does appear to lead to a significant increase in the fraction of total low angle grain boundaries.

(3) Grain refinement mechanism seems to be due to the dynamic recovery of ferrite and to the continuous dynamic ferrite recrystalization. Identical mechanisms are responsible by the ultrafine grain formation in ARB and ECAP processes.

(4) The results obtained for grain sizes and micorhardness suggest that the Hall-Petch closely describes this relationship.

(5) It seems clear that warm torsion testing is a useful and practical tool for the study of grain refinement mechanisms by means of severe plastic deformation.

\section{REFERENCES}

1) B. Q. Han and S. Yue: J. Mater. Process. Technol., 136 (2003), 100.

2) R. Song, D. Ponge, D. Raabe, J. G. Speer and D. K. Matlock: Mater. Sci. Eng. A, 441 (2006), 1.

3) Y. Fukuda, K. Oh-Ishi, Z. Horita and T. G. Langdon: Acta Mater, 50 (2002), 1359.

4) Y. Ivanisenko, W. Lojkowski, R. Z. Valiev and H.-J. Fecht: Acta Mater, 51 (2003), 5555.

5) Y. Saito, H. Utsunomiya, N. Tsuji and T. Sakai: Acta Mater., 47 (1999), 579.

6) N. Tsuji, Y. Saito, H. Utsunomiya and S. Tanigawa: Scr. Mater, 40 (1999), 795

7) A. L. M. Costa, A. C. C. Reis, L. Kestens and M.S. Andrade: Mater. Sci. Eng. A, 406 (2005), 279.

8) N. Tsuji, Y. Ito, Y. Saito and Y. Minamino: Scr. Mater, 47 (2002), 893.

9) H. Pirgazi, A. Akbarzadeh, R. Petrov and L. Kestens: Mater. Sci. Eng. A, 497 (2008), 132.

10) S. H. Cho, K. B. Kang and J. J. Jonas: ISIJ Int., 41 (2001), 63.

11) S. V. S. Narayana Murty and S. Torizuka: ISIJ Int., 48 (2008), 1088.

12) R. Song, D. Ponge and D. Raabe: Acta Mater, 53 (2005), 4881.

13) R. Song, D. Ponge, D. Raabe and R. Kaspar: Acta Mater., 53 (2005), 845.

14) N. Tsuji, N. Kamikawa, R. Ueji, N. Takata, H. Koyama and D. Terada: ISIJ Int., 48(2008), 1114.

15) B. L. Li, N. Tsuji and N. Kamikawa: Mater. Sci. Eng. A, 423 (2006), 331.

16) S. Lee, Y. Saito, N. Tsuji, H. Utsunomiya and T. Sakai: Scr. Mater, 46 (2002), 281.

17) G. Krallics and J. G. Lenard: J. Mater. Process. Technol., 152 (2004), 154.

18) B. Cherukuri, T. S. Nedkova and R. Srinivasan: Mater. Sci. Eng. A, 410 (2005), 394.

19) Y. Tomota, A. Narui and N. Tsuchida: ISIJ Int., 48 (2008), 1107. 